
\title{
25 Research Soure \\ Effect of Pulsed Ultrasound on Plasma Morphology and Its Changing Mechanism
}

\section{Huijing Zhang}

School of Mechatronics Engineering, Northeast Forestry University, Harbin 150040, China

\section{Fan Qing-Kai}

State Key Laboratory of Advanced Welding and Joining, Harbin Institute of Technology, Harbin, 150001, China

\section{Chenglei Fan}

State Key Laboratory of Advanced Welding and Joining, Harbin Institute of Technology, Harbin, 150001, China

Chen Chao ( $\nabla$ chaochenaw@126.com )

School of Mechatronics Engineering, Northeast Forestry University, Harbin 150040, China

\section{Research Article}

Keywords: Pulsed ultrasound, TIG, Plasma, Acoustic streaming

Posted Date: April 7th, 2021

DOl: https://doi.org/10.21203/rs.3.rs-376925/v1

License: (c) (i) This work is licensed under a Creative Commons Attribution 4.0 International License.

Read Full License 


\section{Effect of Pulsed Ultrasound on Plasma Morphology and Its Changing Mechanism}

Huijing Zhang ${ }^{1}$, Fan Qing-Kai ${ }^{2}$, Chenglei Fan ${ }^{2}$, Chen Chao ${ }^{1,2 *}$

1.School of Mechatronics Engineering, Northeast Forestry University, Harbin 150040, China

2.State Key Laboratory of Advanced Welding and Joining, Harbin Institute of Technology, Harbin, 150001, China

*Corresponding author: Dr. Chao Chen, Tel. +86-15776849120, Fax. +86- 045182190397. E-mail address: chaochenaw @126.com

Postal address: School of Mechatronics Engineering, Northeast Forestry University, No.26 Hexing Road, Harbin, China, 150040 


\section{Effect of Pulsed Ultrasound on Plasma Morphology and Its Changing Mechanism}

Abstract: Arc plasma shape under pulsed and continuous ultrasound field were studied in this research using self-developed welding device which combines arc and ultrasound field coaxially. The results show that, compared with the arc of conventional tungsten inert gas welding, the shape of arc under pulsed ultrasound field relate to the pulse frequency. From $1 \mathrm{~Hz}$ to $20 \mathrm{~Hz}$, the arc plasma expands and contracts periodically in one pulse. When more than $20 \mathrm{~Hz}$, the arc plasma contracts as the pulse frequency increases. During high pulse frequency, the arc shape become steady and similar to those in continuous ultrasound field. When in $500 \mathrm{~Hz}$, the contraction ratio of arc projected area under pulsed ultrasound field reaches $38 \%$, comparing with $30 \%$ of the same power continuous ultrasound field, i.e., in high frequency, low power pulsed ultrasound can obtain arc control effect similar to high power continuous ultrasound, raising ultrasound energy efficiency. The mechanism of ultrasonic influence on arc is analyzed based on sound pressure and acoustic streaming. Keywords: Pulsed ultrasound; TIG; Plasma; Acoustic streaming 1 Introduction

In stainless steel and non-ferrous metal welding, tungsten inert gas welding (TIG) has the characteristics of stable welding process, good protection and satisfactory weld bead formation, which is one of the most widely used welding methods ${ }^{[1-3]}$. Researchers 
are committed to developing high-efficiency TIG, developing various TIG welding methods such as pulsed current TIG ${ }^{[4]}$, magnetic control TIG ${ }^{[5]}$, laser-TIG hybrid welding ${ }^{[6]}$, twin-electrode TIG ${ }^{[7]}$, activated flux TIG ${ }^{[8]}$ and so on. As a new welding and arc control method, ultrasonic assisted arc welding has the advantages of noncontact, no electrical interference, controllable parameters and easy adjustment. It has gradually become a research hotspot. Ultrasound is used to improve arc stability in underwater welding ${ }^{[9]}$, to suppress hot cracks in superalloy welding ${ }^{[10]}$ and to reduce the formation of Laves phase in nickel alloy welding ${ }^{[11]}$. In general, the impact of ultrasound refines grain size in weld seam (especially columnar grain in fusion line) ${ }^{[12]}$, increases weld penetration ${ }^{[13]}$, promotes uniform composition ${ }^{[14]}$.

Professor Yang Chunli of Harbin Institute of Technology first realized the coaxial composite of ultrasound and arc ${ }^{[13]}$. Experiments showed that when using $30 \mathrm{~A}$ welding current, the force of hybrid arc is more than twice the conventional TIG arc pressure. The arc force distribution transforms from a double-sided exponential function to a close Gaussian function, which favor deep penetration and high productivity ${ }^{[15]}$. The double exposure holographic interferometry also verified that the arc contracts under ultrasound field: the central energy density of the arc is more concentrated and the arc temperature increases ${ }^{[16]}$. Added ultrasonic to the TIG process, the weld grains vary from columnar shape to equiaxed ${ }^{[12]}$, and the weld porosity is reduced ${ }^{[17]}$. The main influencing factors of the ultrasound field distribution are the height of the radiator, the shape of the radiator, the diameter of the radiator and the frequency of ultrasound. When the height of the radiator is an integer multiple of the half wavelength of the ultrasonic wave, a standing wave sound field is formed, and the acoustic energy intensity reaches maximum ${ }^{[18]}$. When the ultrasonic frequency and the output amplitude increase, the sound pressure amplitude increases. When the shape of radiator changes from a plane to a concave surface, the acoustic pressure increases and becomes more concentrated [19].

However, researches reported mostly focus on the design of hybrid welding equipment and the phenomenological rule of continuous ultrasound-assisted TIG (UTIG), lack of in-depth study of the mechanism between ultrasound and arc plasma. In 
order to utilize ultrasonic efficiently and better regulate the welding process, this paper proposes pulsed ultrasound assisted tungsten inert gas welding (PU-TIG). The morphology of arc plasma in pulsed ultrasound field are studied in this paper, which provides a reference for developing ultrasound-arc hybrid welding.

\section{Materials and methods}

\subsection{Equipment}

German LORCH V24 TIG welding power was used in this study and work in DCRP mode to obtain stable arc. The ultrasonic system uses the commercial HDF01 intelligent ultrasonic generator. The generator can output square wave or continuous waves, with maximum output power of $3000 \mathrm{~W}$. The ultrasonic frequency is $20 \mathrm{kHz}$. The self-designed ultrasonic-arc hybrid torch uses transducer (made by series piezoelectric ceramics) to convert pulse current of the ultrasonic generator into mechanical vibration. The mechanical vibration is amplified by the stepped horn and then emits to the arc plasma zone. The long tungsten pole passes through the hole in the center of the horn. The schematic diagram of the study equipment and the physical diagram of the composite torch are shown in Fig.1 (a) and (b).

(a)

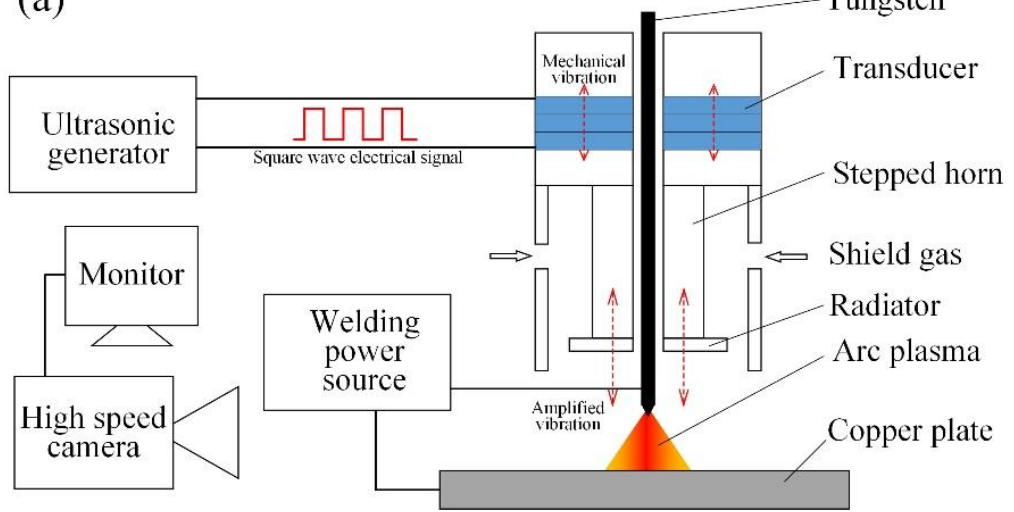

(b)

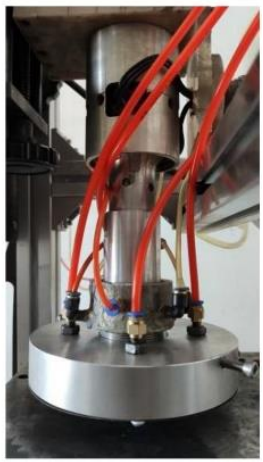

Fig.1. Working way of the system. (a) diagrammatic sketch; (b) hybrid welding torch.

In this study, the copper plate acts as anode. The shielding gas is $99.99 \%$ argon. The peak power of pulsed ultrasound is $2100 \mathrm{~W}$, the background power is $900 \mathrm{~W}$, the frequency ranges from $0 \mathrm{~Hz}$ to $500 \mathrm{~Hz}$, and the duty cycle is $50 \%$. Other welding parameters are shown in Table 1.

Table 1. Welding parameters. 
weld current height of tungsten angle of tungsten shielding gas flow height of acoustic

\begin{tabular}{ccccc}
$(I / \mathrm{A})$ & $(\mathrm{l} / \mathrm{mm})$ & $\left(\alpha /{ }^{\circ}\right)$ & $\left(\mathrm{V} / \mathrm{L} \cdot \mathrm{min}^{-1}\right)$ & $(H / \mathrm{mm})$ \\
\hline 70 & 3 & 45 & 15 & 12
\end{tabular}

High-speed cameras Phantom V341 by American Vision Research Corporation was used to record arc image. The exposure parameter of the camera with filter was set as 2000 frames and exposure time $60 \mu$ s.

\subsection{Arc shape study conditions}

The arc image obtained by high-speed camera are processed as shown in Fig.2. Simplify the image and extract necessary parameters to describe the arc shape. The arc projected area is defined as the number of bright pixels.
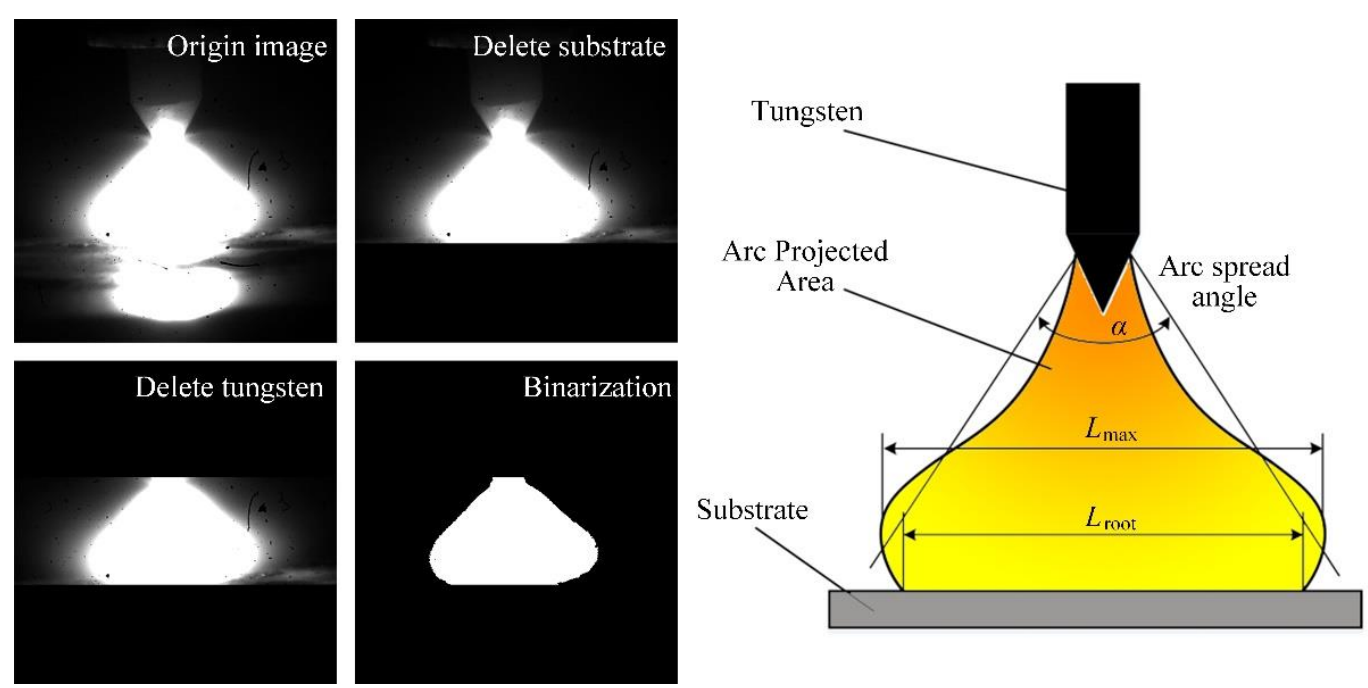

Fig.2. Arc image processing and parameter extracted from image

In order to study the influence of ultrasound on the arc plasma, several geometric parameters are defined:

$$
\begin{gathered}
\Delta S=\frac{S_{\mathrm{TIG}}-S_{\mathrm{U}}}{S_{\mathrm{TIG}}} \cdot 100 \% \\
\Delta L_{\text {max }}=\frac{L_{\text {max-TIG }}-L_{\text {max }-\mathrm{U}}}{L_{\text {max }-\mathrm{TIG}}} \cdot 100 \% \\
\Delta L_{\text {root }}=\frac{L_{\text {root-TIG }}-L_{\text {root}-\mathrm{U}}}{L_{\text {root}-\mathrm{TIG}}} \cdot 100 \%
\end{gathered}
$$

where $\Delta S$ is the change rate of arc projected area, $S_{\text {TIG }}$ is area of TIG arc, $S_{\mathrm{U}}$ is arc area of U-TIG or PU-TIG, $\Delta L_{\max }$ is change rate of maximum arc width, $L_{\text {max-TIG }}$ is maximum arc width of TIG, and $L_{\max -\mathrm{U}}$ is maximum arc width of U-TIG or PU-TIG, 
$\Delta L_{\text {root }}$ is change rate of bottom arc width, $L_{\text {root-TIG }}$ is bottom arc width of TIG, and $L_{\text {root- }}$ $\mathrm{U}$ is bottom arc width of U-TIG or PU-TIG.

\section{Results}

\subsection{Influence of ultrasonic power on arc behavior}

Arc images of TIG and different ultrasonic power U-TIG are shown in Fig.3. In the welding current of $70 \mathrm{~A}$, the arc exhibits different degrees of contraction under continuous ultrasound. As the ultrasonic power increases, the arc shows more shrinkage, which is manifested by a reduction in arc width and a decrease in arc spread angle. Comparing the arc images of U-TIG of different welding currents, the degree of shrinkage reduced as the welding current increases. This is because arc with a large current have the ability to resist external disturbances.

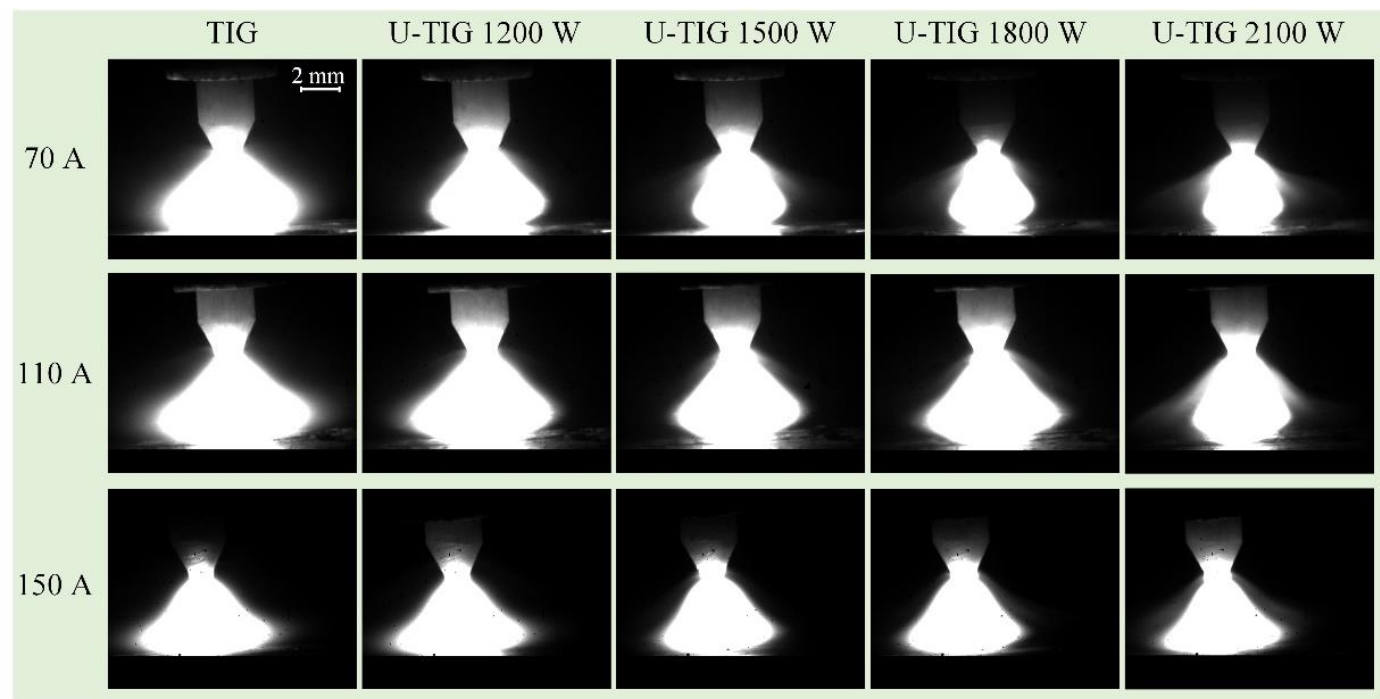

Fig.3. Influence of ultrasonic power on U-TIG arc behavior.

\subsection{Influence of pulse frequency on PU-TIG arc behavior}

The PU-TIG arc images of different ultrasonic pulse frequencies are shown in Fig.4. In low pulse frequency (for example, less than $20 \mathrm{~Hz}$ ), the arc shape remain unchanged during the period of the ultrasonic peak and background value. During the peak value, the arc shrinks, which is manifested by a significant decrease in the maximum arc width. 


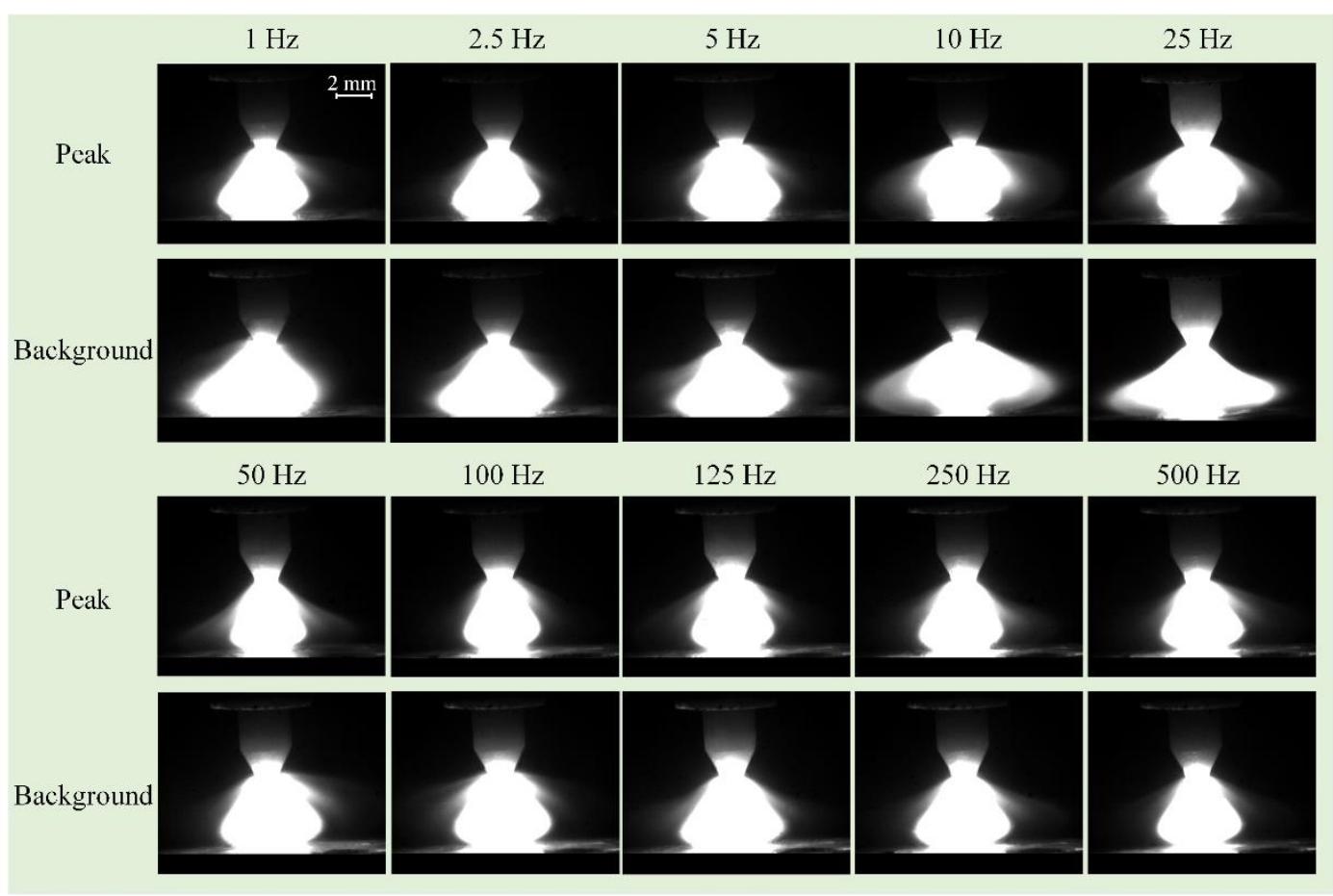

Fig.4. Influence of pulse frequency on PU-TIG arc behavior.

At the pulse frequency of $10 \mathrm{~Hz}$, arc shape in one cycle are shown in Fig.5. In 0.1 $\mathrm{s} \sim 0.2 \mathrm{~s}$ and $0.9 \mathrm{~s} \sim 1.0 \mathrm{~s}$, the arc is stable and diffused. In $0.4 \mathrm{~s} \sim 0.7 \mathrm{~s}$, the arc is stable and contracted. At about $0.3 \mathrm{~s}$ and $0.8 \mathrm{~s}$, the ultrasonic output power changes abruptly, while the arc shape are in a transitional period.

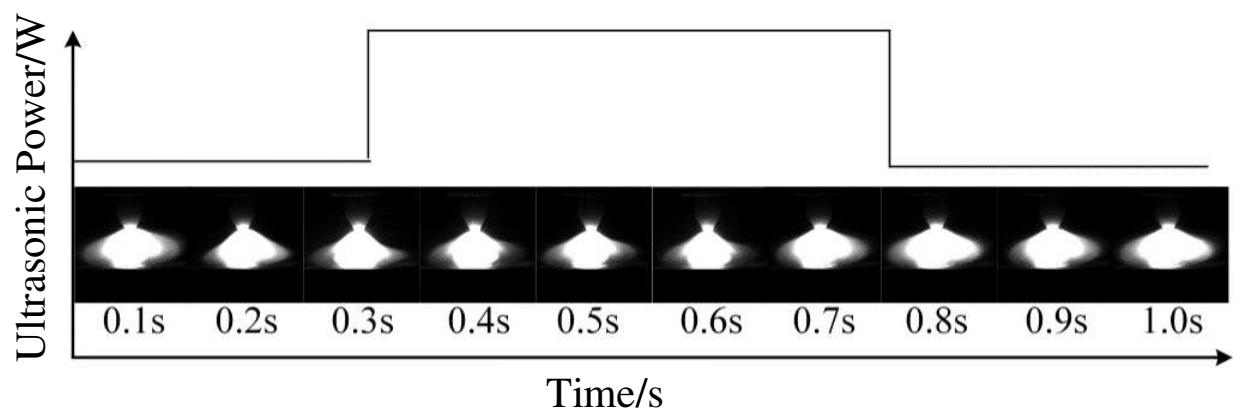

Fig.5 Arc shape during one pulse cycle.

As the pulse frequency increases, although the arc still exhibits the same periodic jitter as the ultrasonic frequency, the arc shape change cannot keep up with the ultrasonic pulse period. In high frequency, arc shape between the background value and peak value tends to be consistent.

In order to quantify the trend of this change, multiple geometric parameters are defined as Eq.(1) to (3), and the results are shown in Fig.8. from the $1 \mathrm{~Hz}$ to $500 \mathrm{~Hz}$, 
arc shrinks more significantly in peak value compared with background value. And as the pulse frequency increases, the arc area change at the peak value stage is small, and the arc area changes greatly at the base value stage. The arc spread angle PU-TIG is overall smaller than TIG.
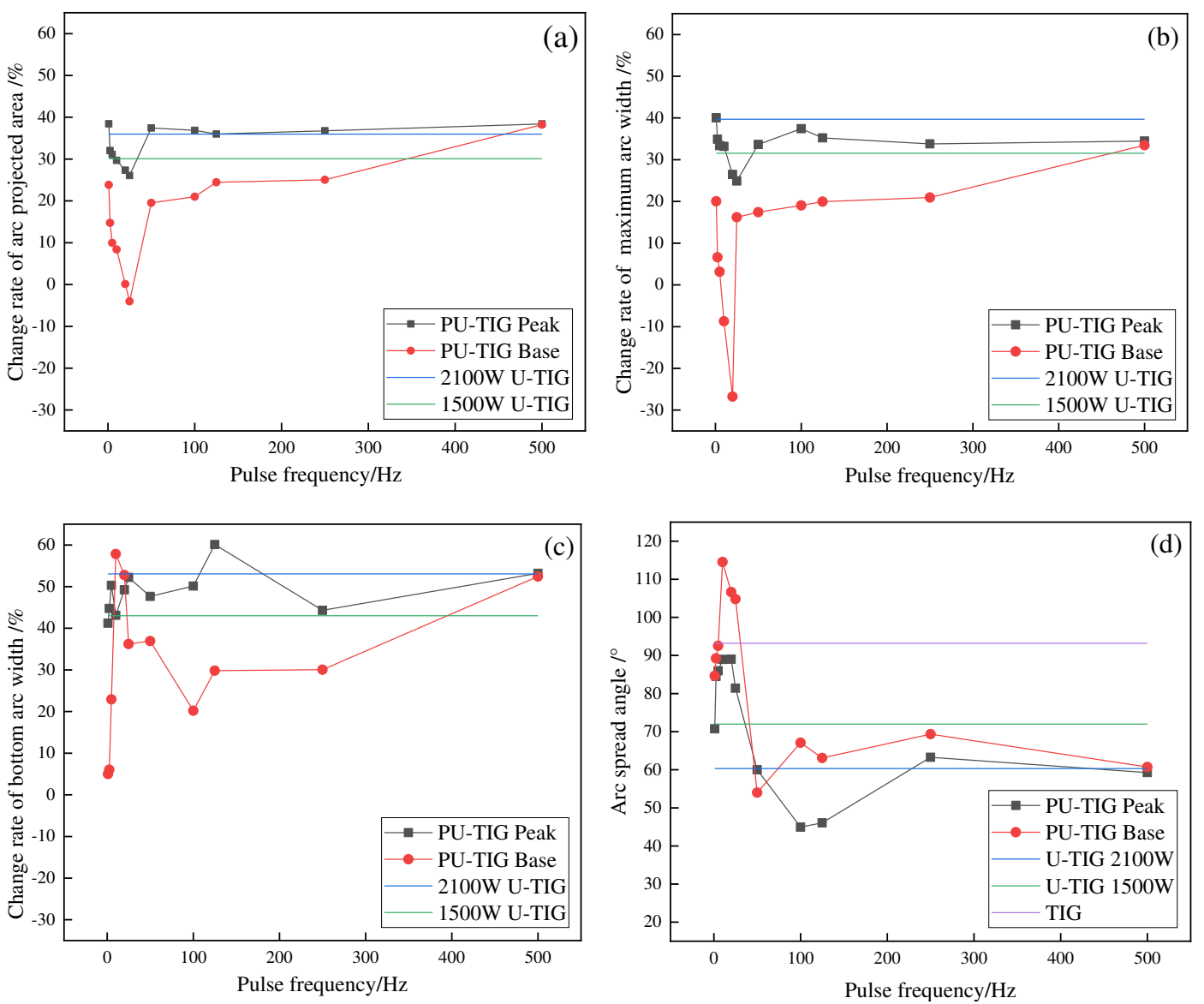

Fig.6. Effect of Pulse Frequency on PU-TIG Arc Shape. (a) change rate of arc projected area; (b) change rate of maximum arc width; (c) change rate of bottom arc width; (d) arc spread angle

It can be seen from the above that in the high pulse frequency, the effect of pulsed ultrasound on the arc shape is nearly consistent with continuous ultrasound. Fig.7 is a comparison of PU-TIG and U-TIG arc shape. The average power of pulsed ultrasound is $1500 \mathrm{~W}$. At $500 \mathrm{~Hz}$, the arc shape of PU-TIG approaches the contraction mode at peak value. That is, increasing the ultrasonic pulse frequency allows the PU-TIG with lower average ultrasonic power to obtain an arc shape similar to those of high power U-TIG. 


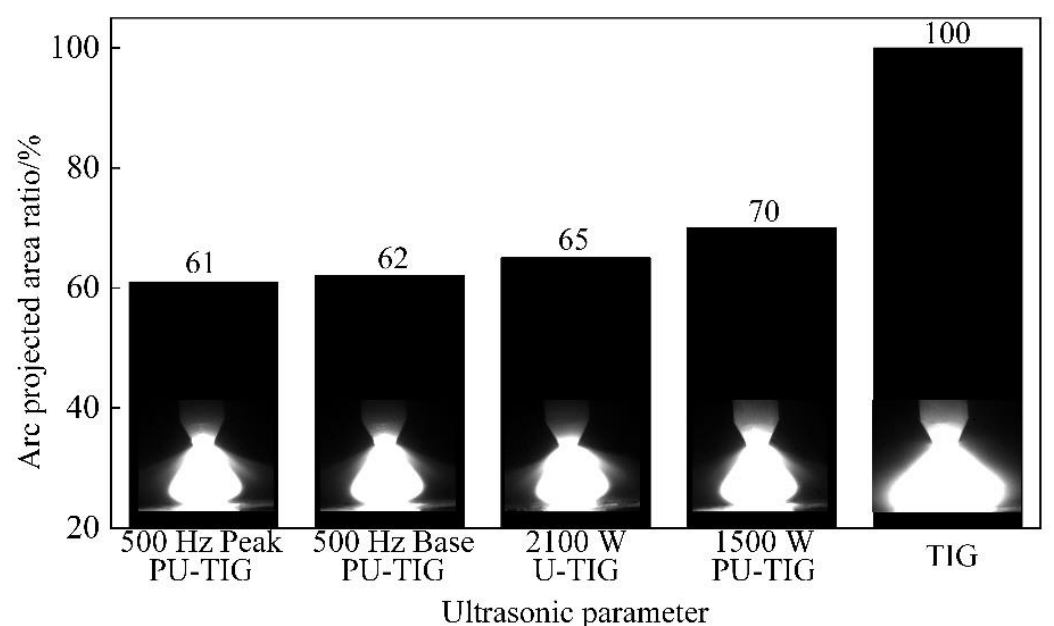

Fig.7. Arc projected area comparison.

\section{2}

\section{Analysis and discussion}

\subsection{Effect of acoustic standing wave on plasma}

When the distance between the radiator and reflector is an integer multiple of the half-wavelength of the sound wave, an acoustic standing wave flied is created ${ }^{[20]}$. To consider the case of plane simple harmonic wave propagating along the $\mathrm{x}$-axis. The sound pressure of the incident wave is $p_{i}$, and the reflected sound pressure is $p_{r}$. The frequency of the incident wave is same as the reflected wave, while the propagating direction is opposite. This two wave interfere and form a synthetic sound pressure $p$, which can be described with mathematical expression ${ }^{[21]}$ :

$$
\begin{gathered}
p_{i}=p_{i m} \mathrm{e}^{\mathrm{j}(\omega t-k x)} \\
p_{r}=p_{r m} \mathrm{e}^{\mathrm{j}(\omega t+k x)} \\
p=p_{i}+p_{r}=2 p_{r m} \cos k x \mathrm{e}^{\mathrm{j} \omega t}+\left(p_{i m}-p_{r m}\right) \mathrm{e}^{\mathrm{j}(\omega t-k x)}
\end{gathered}
$$

where $p_{i m}$ and $p_{r m}$ are the amplitude of sound pressure of incident wave and the reflected wave respectively, $\omega=2 \pi f$ is circular frequency, $t$ is time, $k=1 / \lambda$ is wave number. From Eq.6, at $x=\mathrm{n} \lambda / 2 \quad(\mathrm{n}=1,2, \cdots)$, the sound pressure reaches maximum; at the $x=(2 \mathrm{n}-1) \lambda / 4 \quad(\mathrm{n}=1,2, \cdots)$, the sound pressure is minimum.

In high-pressure condition, arc contracts and transform from diffused bell shape to compact cylinder shape ${ }^{[22]}$. The arc voltage increase as the ambient pressure rises and becomes brighter ${ }^{[23]}$. The Saha's equation can be used to describe thermal ionization [24]: 


$$
\frac{\alpha^{2}}{1-\alpha^{2}} \cdot p=\frac{2 z_{+}}{z}\left[\frac{2 \pi m_{\mathrm{e}}}{h^{2}}\right]^{3 / 2}(k T)^{5 / 2} \exp \left[-\frac{e U_{\mathrm{i}}}{k T}\right]
$$

where $\alpha$ is the degree of ionization of plasma, $p$ is ambient pressure, $z_{+}$and $z$ is internal partition function of ion and atom respectively, which describe the sum of possible energy states, $m_{\mathrm{e}}$ is the mass of electron, $k$ is Boltzmann's constant, $h$ is Planck's constant, $T$ is the thermodynamic temperature, $e U_{\mathrm{i}}$ is the energy of the $\mathrm{i}$-th energy level. When the degree of ionization is low and the temperature $T$ is constant, the relationship

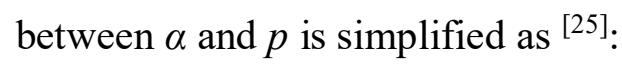

$$
\alpha \propto \frac{1}{p^{1 / 2}}
$$

With the increase of pressure, the degree of ionization decreases, i.e., the conductivity of the plasma decreases ${ }^{[26]}$. In order to maintain the set welding current, the drive_ arc voltage must rise, which has been proved by measurement ${ }^{[22][23] ~[27]}$. The arc plasma must contract to increase the ion density to maintain conductivity.

\subsubsection{Effect of acoustic streaming on plasma}

When the acoustic energy is large enough, the density of medium substituted into the Navier-Stokes equations should be a function of pressure instead of a fixed value ${ }^{[28]}$. It leads to a non-zero time averaged pressure, which is the so-called acoustic radiation pressure ${ }^{[20]}$. The value of acoustic radiation pressure is proportional to the energy density of sound wave ${ }^{[29]}$. If the medium is viscous or has boundaries, the energy of the sound wave dissipates and thereby acoustic radiation pressure difference forms. When acoustic radiation pressure difference acts on solid particles, it is called acoustic radiation force, which is used in acoustic suspension ${ }^{[20][30]}$. It cause the medium to flow when act on the the medium itself___acoustic streaming [31]. Rayleigh firstly studied the acoustic streaming phenomenon between two parallel plates in the air ${ }^{[32]}$. Two clockwise and two counter clockwise circulations are formed between parallel plates as shown in Fig.8 (a). 


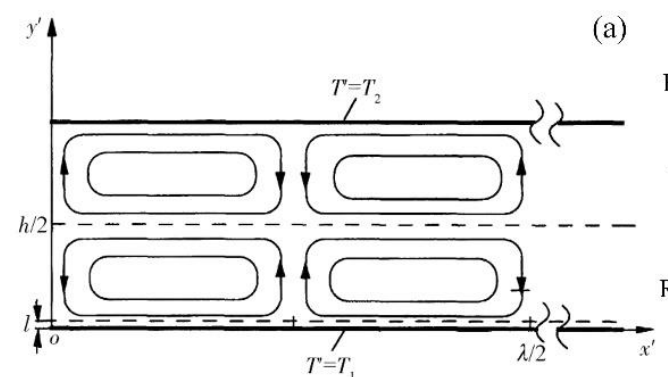

(a)

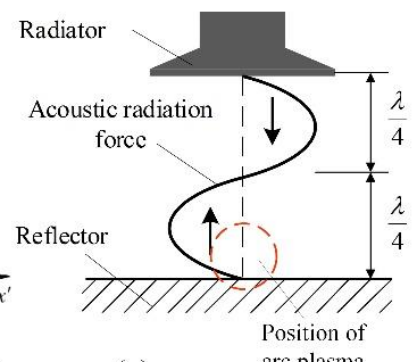

(c)

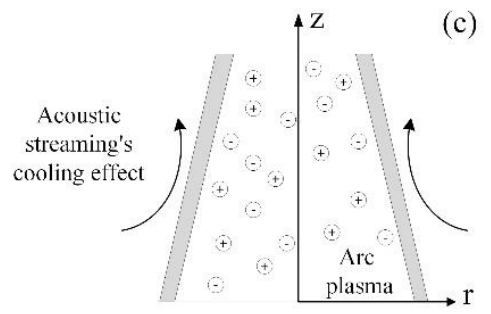

Fig. 8 Mechanism of acoustic streaming and its effect on arc. (a) Rayleigh's acoustic streaming in a standing wave field ${ }^{[32]}$; (b) distribution of acoustic radiation force; (c) cooling effect of acoustic streaming on arc;

The energy of the acoustic streaming created by ultrasound is sufficient for drying ${ }^{[33]}$ and melt processing ${ }^{[34]}$. When acoustic streaming acts on the arc, it is equivalent to add a forced-cooling convection. It blows away the low temperature neutral particles and promote deionization of arc plasma. The heat lost in the form of radiation also increases under high pressure ${ }^{[35]}$. Based on the principle of minimum voltage ${ }^{[36]}$, when the heat loss of the arc increases, in order to maintain the balance of heat production and heat dissipation, the arc contract to reduce the surface area and thus reduce the heat dissipation. In other word, cooling limits the range of ions and confines them to smaller zone. When the density of particles increases, it is easier for electrons to transferred its energy to neutral particles and ions through collision ${ }^{[37]}$. For the temperature of the electron is higher than other particles, temperature homogenization means increase in temperature of the arc, which was proved by holographic interference measurement ${ }^{[16]}$.

\subsection{Mechanism of ultrasonic on plasma}

From part 3.2, when pulse frequency is more than $250 \mathrm{~Hz}$, the arc area of pulsed ultrasound is smaller than that of un-pulsed ultrasound. It can be explained by the magnetic contraction of arc. According to right hand grip rule, the wires conducting the same direction current attract each other. For a cylindrical conductor of fluid, the radial 
pressure is ${ }^{[25]}$ :

$$
P_{r}=K \frac{I}{\pi R^{2}}\left(R^{2}-r^{2}\right)
$$

where $P_{r}$ is the pressure at radius $r, K$ is a constant, $I$ is current, $R$ is radius of the cylindrical conductor.

From Eq.6, the decrease of conductor radius will lead to the increase of contraction force. As a result, the contracted arc should exhibit a more significant magnetic contraction effect. To confirm this suppose, a comparative test was made as shown in Fig.9. It takes $0.044 \mathrm{~s}$ for the arc to shrink to stable and takes $0.266 \mathrm{~s}$ to expand. The speed of arc expansion is lower than that of contraction. Arc cannot expand completely under high-frequency pulse ultrasonic and thus its shape is similar to that of continuous ultrasonic.

Add continuous ultrasound of $1800 \mathrm{~W}$
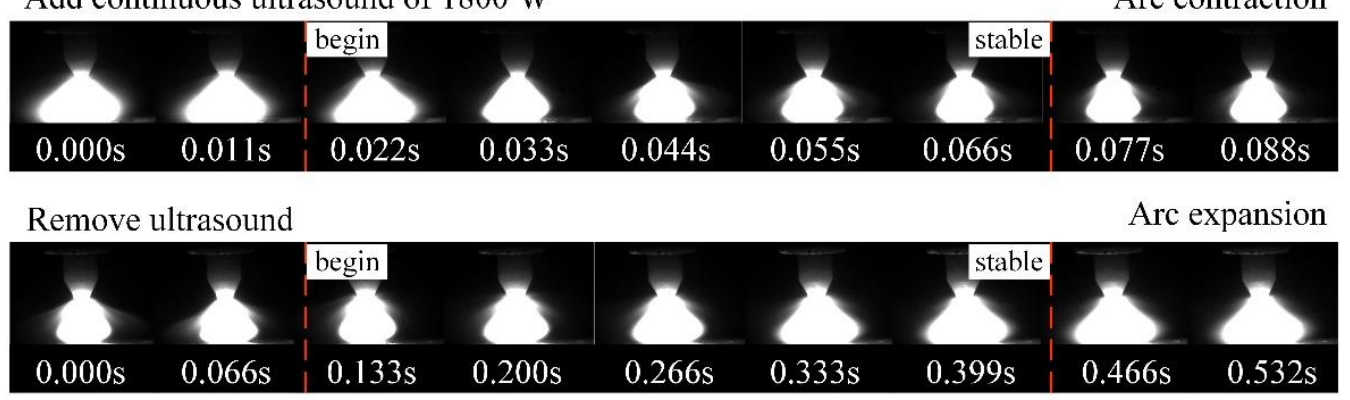

Fig.9. The speed of arc contraction and expansion.

\section{Conclusion}

(1) With the increase of ultrasonic power, the degree of contraction of the arc gradually increases, which is represented by the reduction of arc area, arc width and diffusion angle. As the arc current increases, the contraction of the arc weakens.

(2) At low pulse frequency $(<20 \mathrm{~Hz})$, the arc shape has a relatively stable period. At the peak value of ultrasound, the arc contracts obviously, and the arc expands at the background value; at high pulse frequency $(>250 \mathrm{~Hz})$, the difference of arc shape between peak and background reduces, which tends to contract stably.

(3) At high pulse frequency, lower power pulsed ultrasound can obtain the arc shape similar to higher power continuous ultrasound. The ultrasound utilization is improved. (4) A local high pressure zone and acoustic streaming formed by ultrasound standing 
wave enhance the cooling of arc and make it shrink.

\section{Ethical Approval}

232 Not applicable

\section{Consent to Participate}

234 Not applicable

\section{Consent to Publish}

$236 \quad$ Not applicable

\section{$237 \quad$ Authors Contributions}

238 Not applicable

\section{$239 \quad$ Funding}

$240 \quad$ No funding

\section{Competing Interests}

242 No conflict of interest exists in the submission of this manuscript, and the manuscript is approved

243 by all authors for publication.

\section{$244 \quad$ Availability of data and materials}

$245 \quad$ Not applicable

\section{References}

[1] Aval H J, Farzadi A, Serajzadeh S, Kokabi A H (2009) Theoretical and experimental study of microstructures and weld pool geometry during GTAW of 304 stainless steel. Int J Adv Manuf Tech 42(11):1043-1051

[2] Jang H, Zhang SD (2017) Current status of research and application of titanium alloys at home and abroad. Adv Mater Ind 3:7-10

[3] Nong Q, Xie YD, Jin CY (2013) Research Status and Prospects of Aluminum Alloy Welding Technology. Hot Work Tech 42(9):160-162

[4] Yang MX (2017) Arc Characteristics in Double-Pulsed VP-GTAW for Aluminum Alloy. J Mater Process Tech 243:9-15

[5] Zhang X, Wang ZM, Li YM (2013) Application Research of Longitudinal Magnetic Field in Magnetron Arc Technology. Electr Weld Mac. 43:38-42

[6] Liu L, Wang J, Gang S (2004) Hybrid laser-TIG welding, laser beam welding and gas tungsten arc welding of AZ31B magnesium alloy. Mater Sci Eng 381(1):129-133 
[7] Schwedersky M B, Silva R H G E, Dutra J C, Willms K (2016) Two-dimensional arc stagnation pressure measurements for the double-electrode GTAW process. Sci Tech Weld Join 21(4):275-280 [8] Vidyarthy R S, Dwivedi D K (2016) Activating flux tungsten inert gas welding for enhanced weld penetration. J Manu Proc 22:211-228

[9] Wang JF, Sun QJ , Zhang T, Tao XY, Jin P, Feng JC (2019) Arc stability indexes evaluation of ultrasonic wave-assisted underwater FCAW using electrical signal analysis. Int J Adv Manuf Tech 103:2593-2608

[10] Thavamani R, Balusamy V, Nampoothiri J, Subramanian R, Ravi K R (2017) Mitigation of hot cracking in Inconel 718 superalloy by ultrasonic vibration during gas tungsten arc welding. J Alloy Compd 40:870-878

[11] Hua C, Lu H, Yu C, Lin D, Chen X (2018) Probing Laves phase formation in Ni-alloy during ultrasonic arc-welding with atomistic modelling. Sci Technol Weld Joi 24:305-312

[12] Chen QH, Lin SB, Yang CL, Fan CL, Ge H (2017) Grain fragmentation in ultrasonic-assisted TIG weld of pure aluminum. Ultrason Sonochem 39:403-411

[13] Sun QJ, Lin SB, Yang CL (2008) Arc characteristic of ultrasonic assisted TIG welding. Chin Weld Eng-En 17(4):52-57

\section{J Mech Eng-En 47(4):53-57}

[16] Yao QT (2017) Ultrasonic-assisted welding arc plasma holographic interference research.

Dissertation, Harbin Institute of Technology (in Chinese)

[17] Yuan HR, Lin SB, Yang CL, Fan CL, Wang S (2011) Microstructure and porosity analysis in ultrasonic assisted TIG welding of 2014 aluminum alloy. Chin Weld Eng-En 20(1):39-43

[18] Xie WJ, Teng PF (2014) Lattice Boltzmann Method for Acoustic Suspension Process. Acta. Phys. Sin. 63(16):235-241

[19] Xie WF, Lin SB, Yang CL, Fan CL (2016) Effect of acoustic field parameters on arc acoustic binding during ultrasonic wave-assisted arc welding. Ultrason Sonochem 29:476-484

289 [21] Du GH, Zhu ZM, Gong XF. (2001) Fundamentals of Acoustics. Nanjing University Press, 
Nanjing (in Chinese)

[22] Huang JQ, Xue L, Lv T, Niu H (2001) Arc characteristics of GMA welding in high-pressure air condition. China Weld Eng-En 21(4):26-31

[23] Wu S, Gong S, Gao H (2019) Arc characteristics of GTAW under high pressure. Sadhana-Acad P Eng S 44(3):70

[24] Kannappan D, Bose T K (1977) Transport properties of a two-temperature argon plasma. Phys Fluids 20:1668-1673

[25] Yang C L, Lin S B (2010) Arc Welding Base. Harbin Institute of Technology Press, Harbin (in Chinese)

[26] Bauder U H (1976) Properties of high pressure arc plasma. Appl Phys 9:105-115

[27] Jiang LP, Wang ZH, Jiao XD, Zhou CF, Fang XM, Ma HX (2007) Characteristics of GTAW arc in underwater welding under high-pressure air condition. Trans China Weld Inst 28(6):1-4 (in Chinese)

[28] Bruus H (2011) Acoustofluidics 1: Governing equations in microfluidics. Lab Chip 11(22):3742-3751

[29] Feng R (1999) Ultrasonics Handbook. Nanjing University Press, Nanjing (in Chinese)

[30] Bruus, Henrik (2012) Acoustofluidics 7: The acoustic radiation force on small particles. Lab Chip 12(6):1014

[31] Mitome H (1998) The mechanism of generation of acoustic streaming. Electr Commun Jpn 81(10):1-8

[32] Rayleigh L (1884) On the circulation of air observed in Kundt's tubes, and on some allied acoustical problems. Philo Trans Royal Soc London 175:1-21

[33] Kowalski S J, Pawt Owski A (2015) Intensification of apple drying due to ultrasound enhancement. J Food Eng 156:1-9

[34] Mi J, Tan D, Lee TL (2015) In Situ Synchrotron X-ray Study of Ultrasound Cavitation and Its Effect on Solidification Microstructures. Metall and Materi Trans B. 46:1615-1619

[35] Kopainsky J (1971) Strahlungstransportmechanismus und Transportkoeffizienten im ArHochdruckbogen. Z. Physik 248:417-432

[36] Guo ZY, Zhao WH (1986) Arc and Thermal Plasma. Science Press, Beijing (in Chinese)

[37] Schmidt H P, Speckhofer G (1996) Experimental and theoretical investigation of high-pressure 
320 arcs. I. The cylindrical arc column (two-dimensional modeling). IEEE T Plasma Sci 24(4):1229$321 \quad 1238$ 


\section{Figures}

(a)

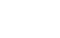

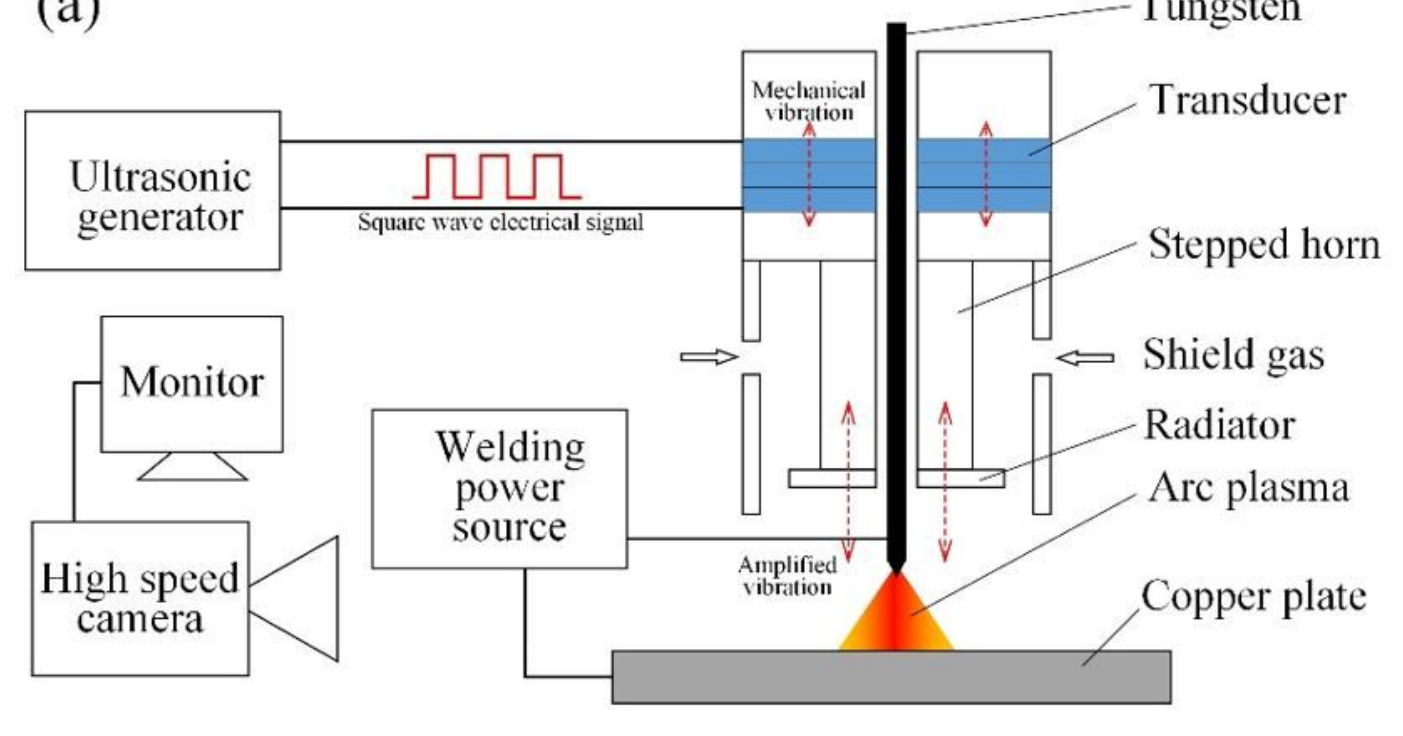

(b)

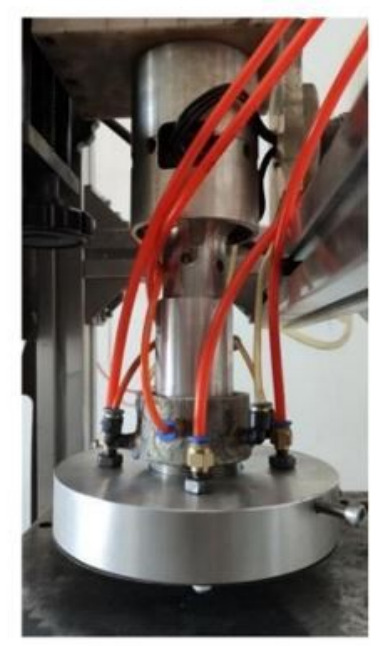

Figure 1

Working way of the system. (a) diagrammatic sketch; (b) hybrid welding torch.
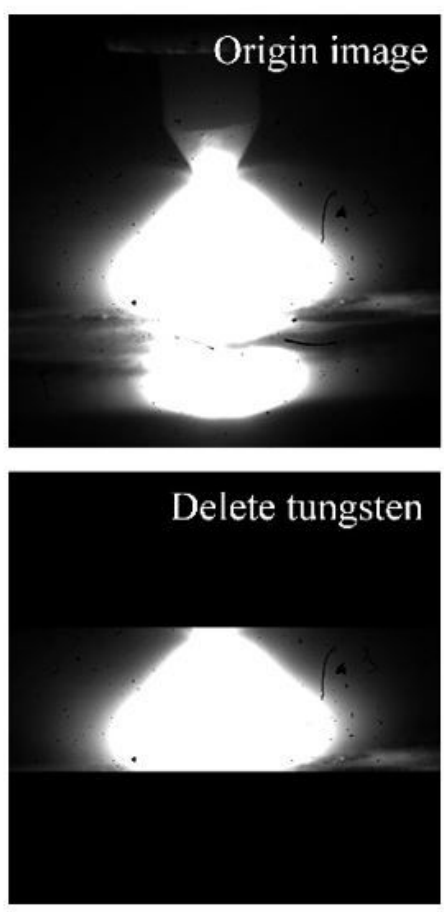
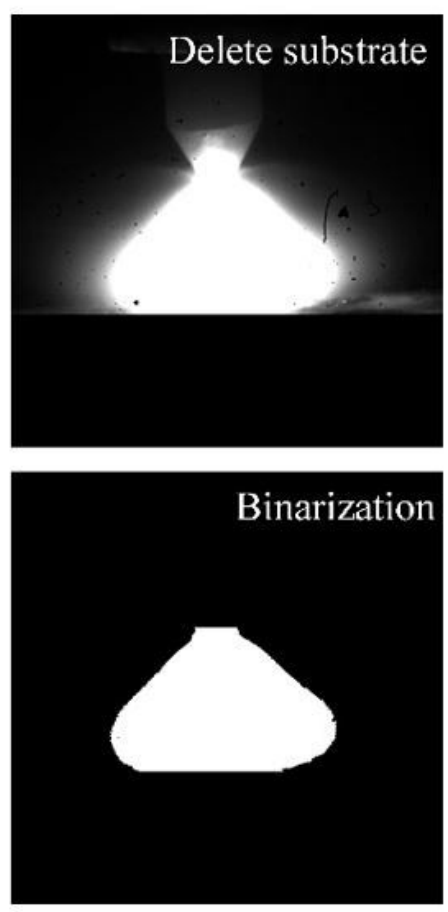

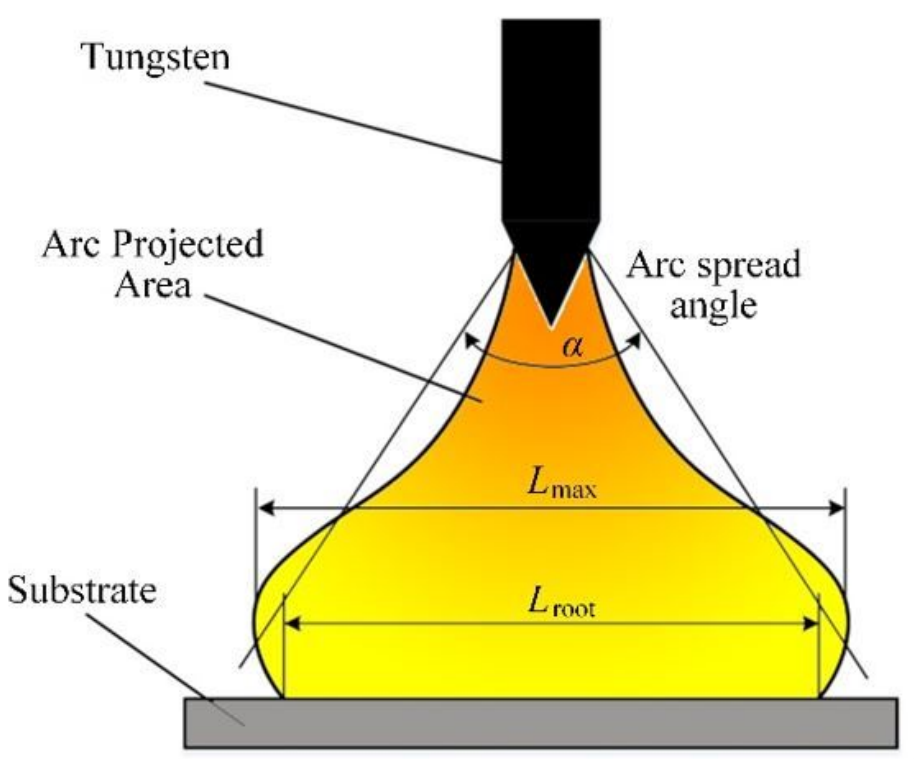

Figure 2

Arc image processing and parameter extracted from image 


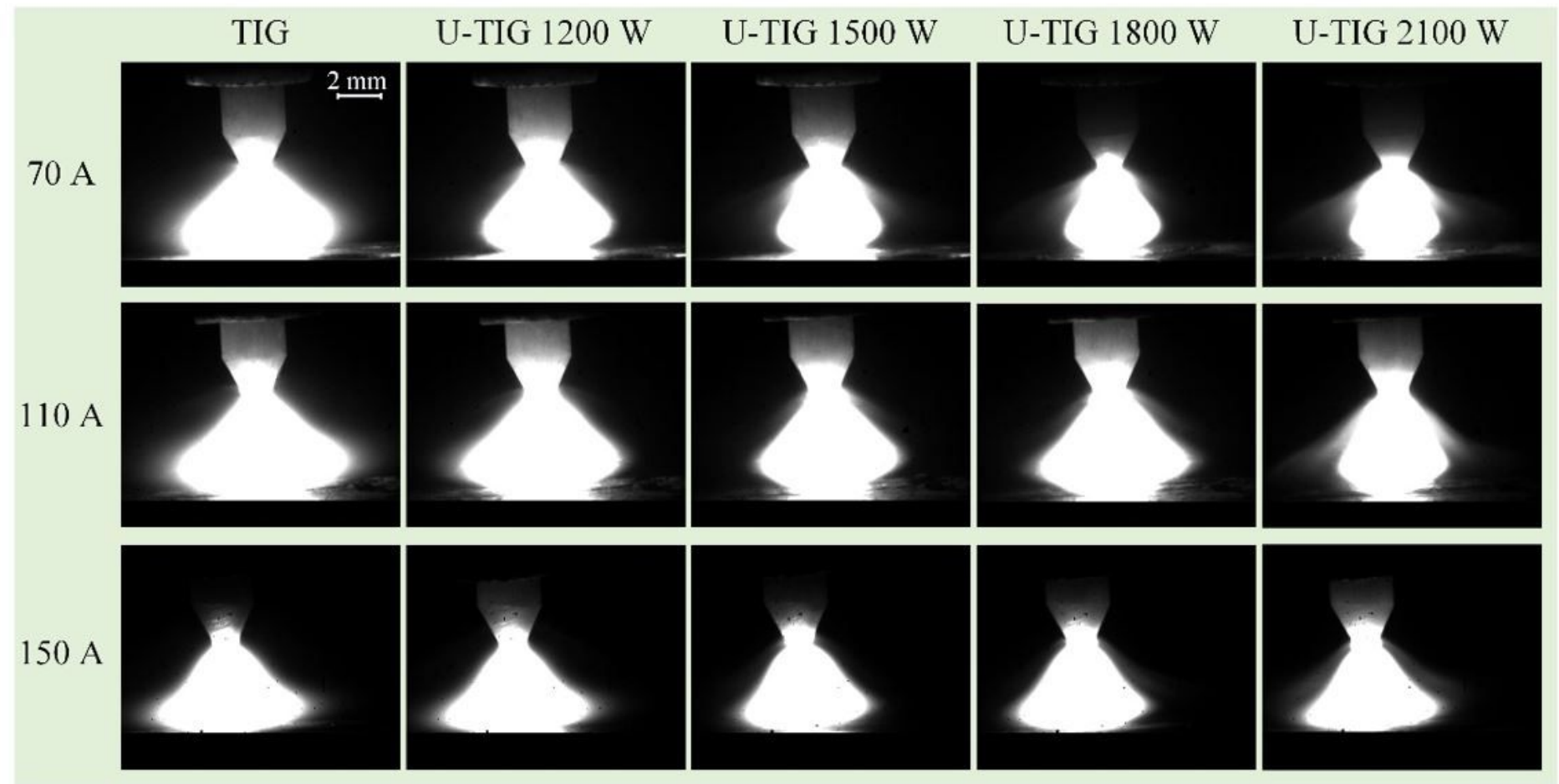

Figure 3

Influence of ultrasonic power on U-TIG arc behavior.

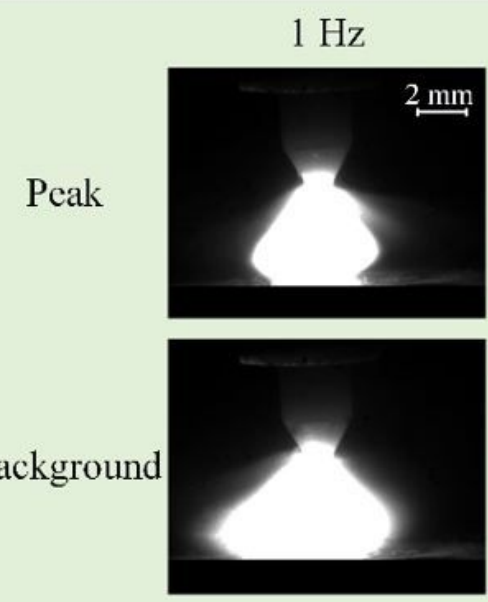

$50 \mathrm{~Hz}$

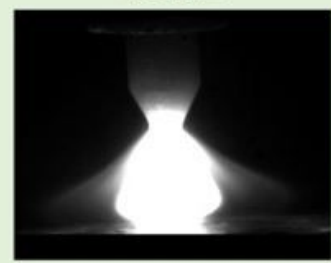

Peak

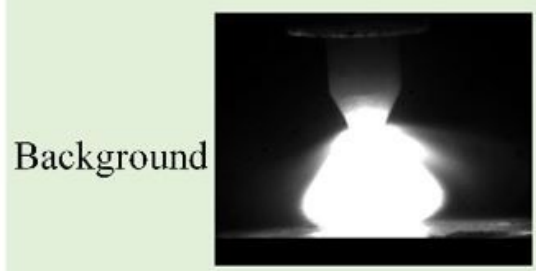

$2.5 \mathrm{~Hz}$
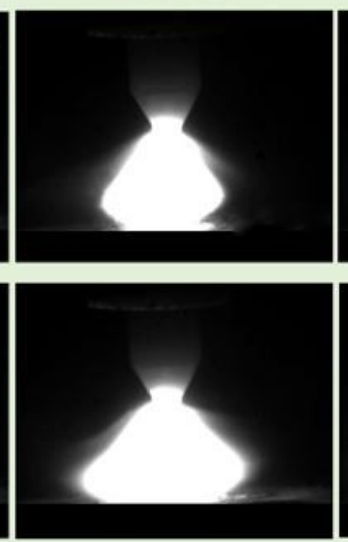

$100 \mathrm{~Hz}$
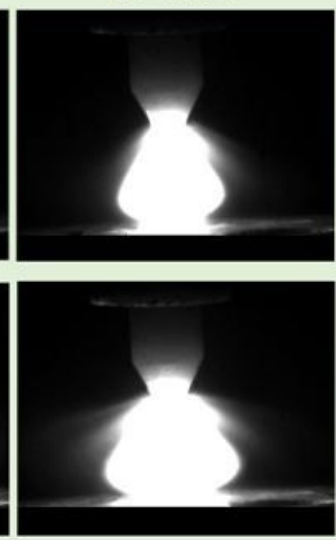

$5 \mathrm{~Hz}$
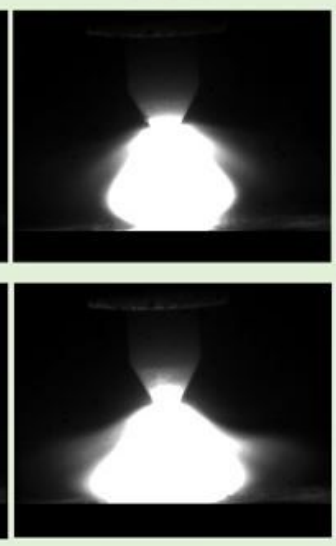

$125 \mathrm{~Hz}$
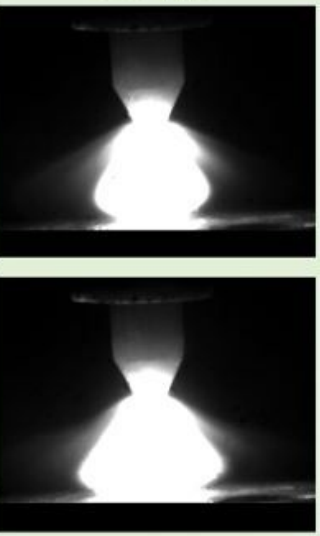

$10 \mathrm{~Hz}$
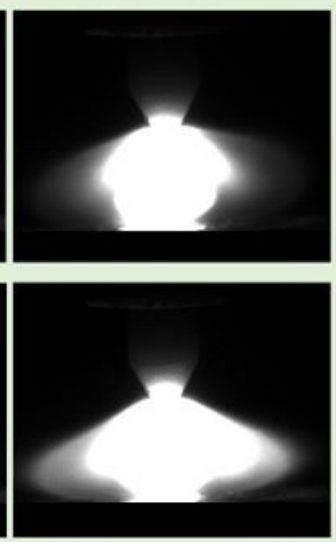

$250 \mathrm{~Hz}$
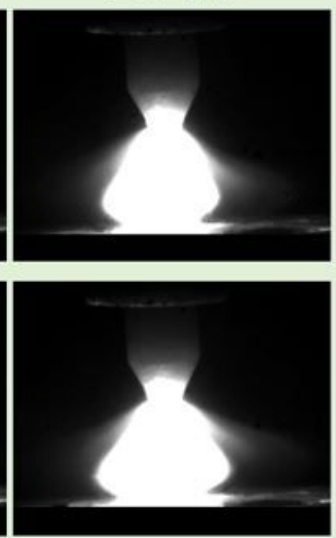

$25 \mathrm{~Hz}$
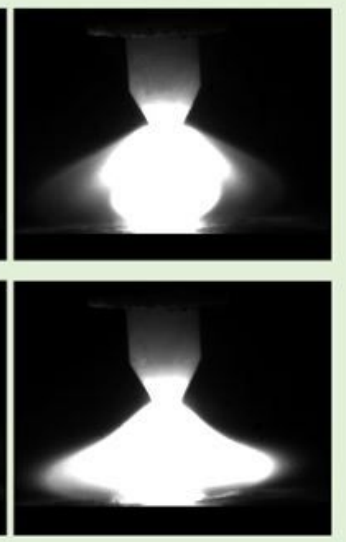

$500 \mathrm{~Hz}$
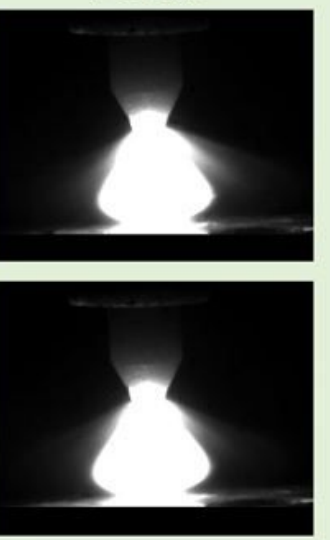


\section{Figure 4}

Influence of pulse frequency on PU-TIG arc behavior.

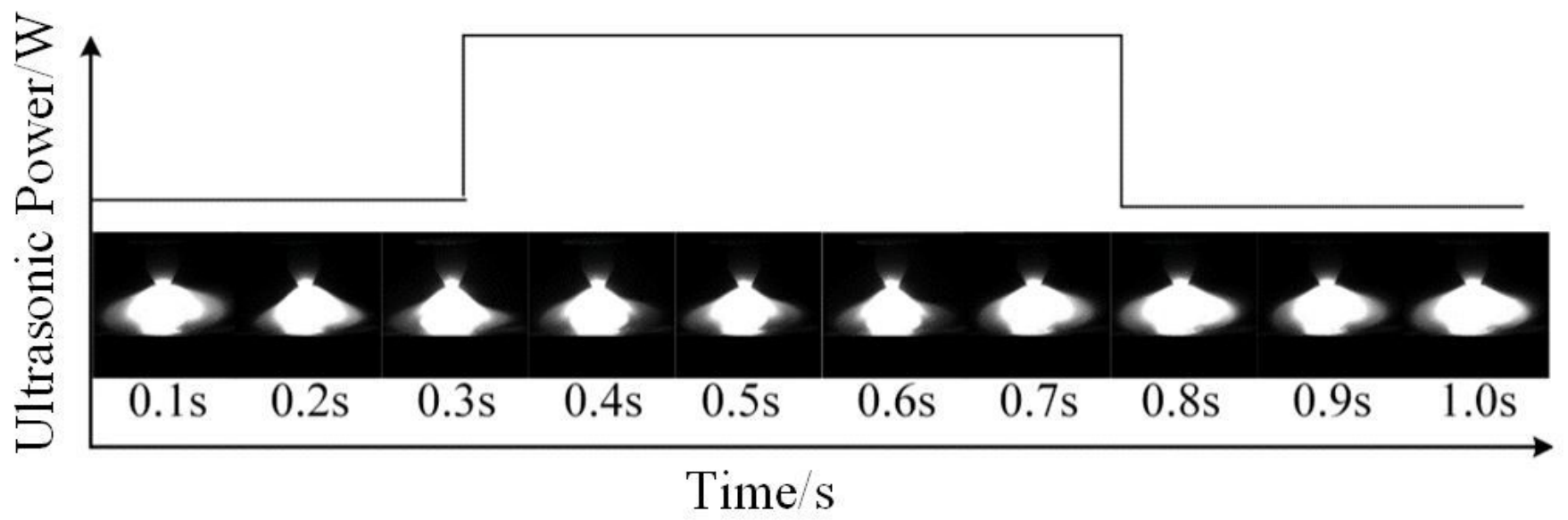

Figure 5

Arc shape during one pulse cycle. 

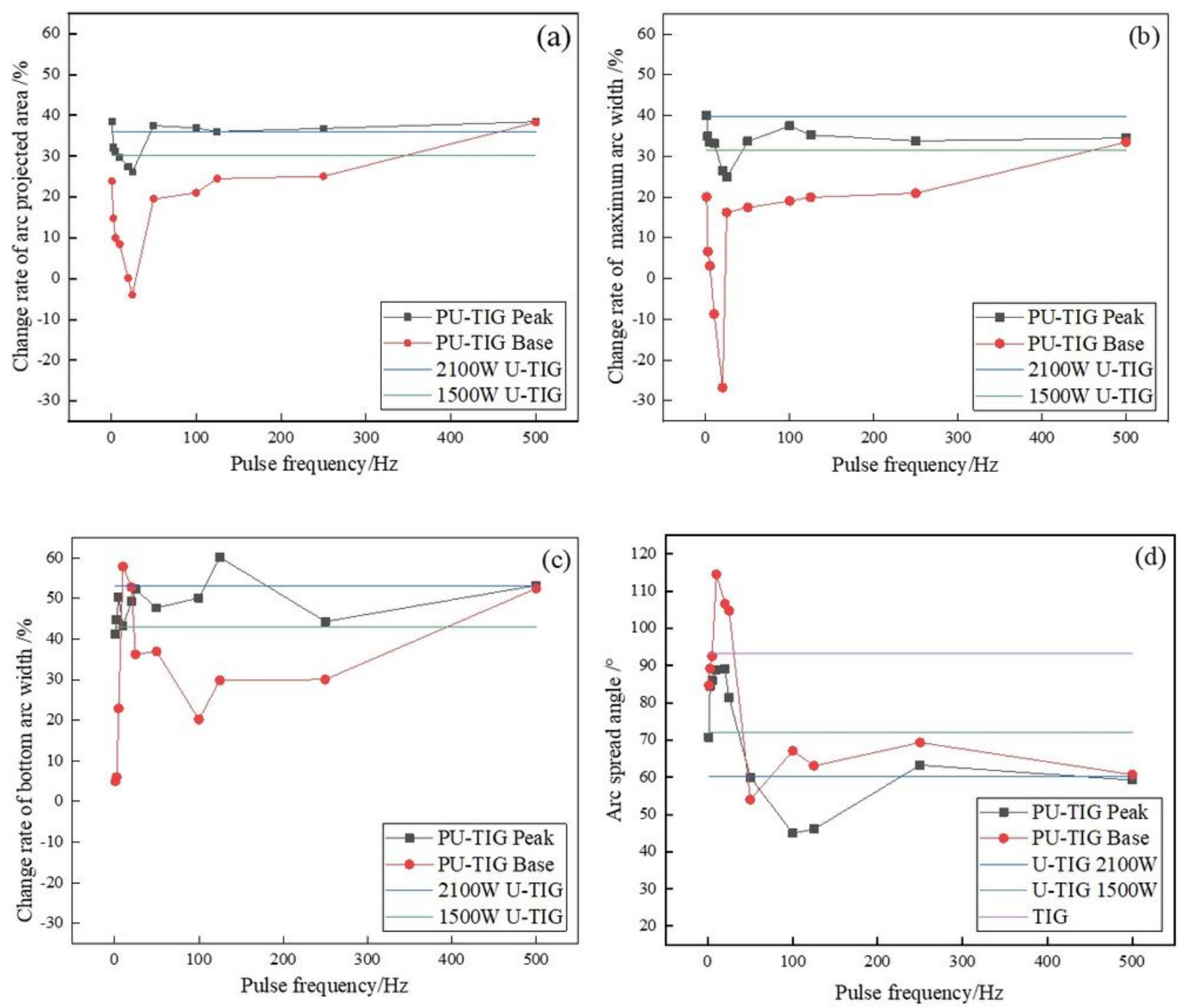

Figure 6

Effect of Pulse Frequency on PU-TIG Arc Shape. (a) change rate of arc projected area; (b) change rate of maximum arc width; (c) change rate of bottom arc width; (d) arc spread angle 


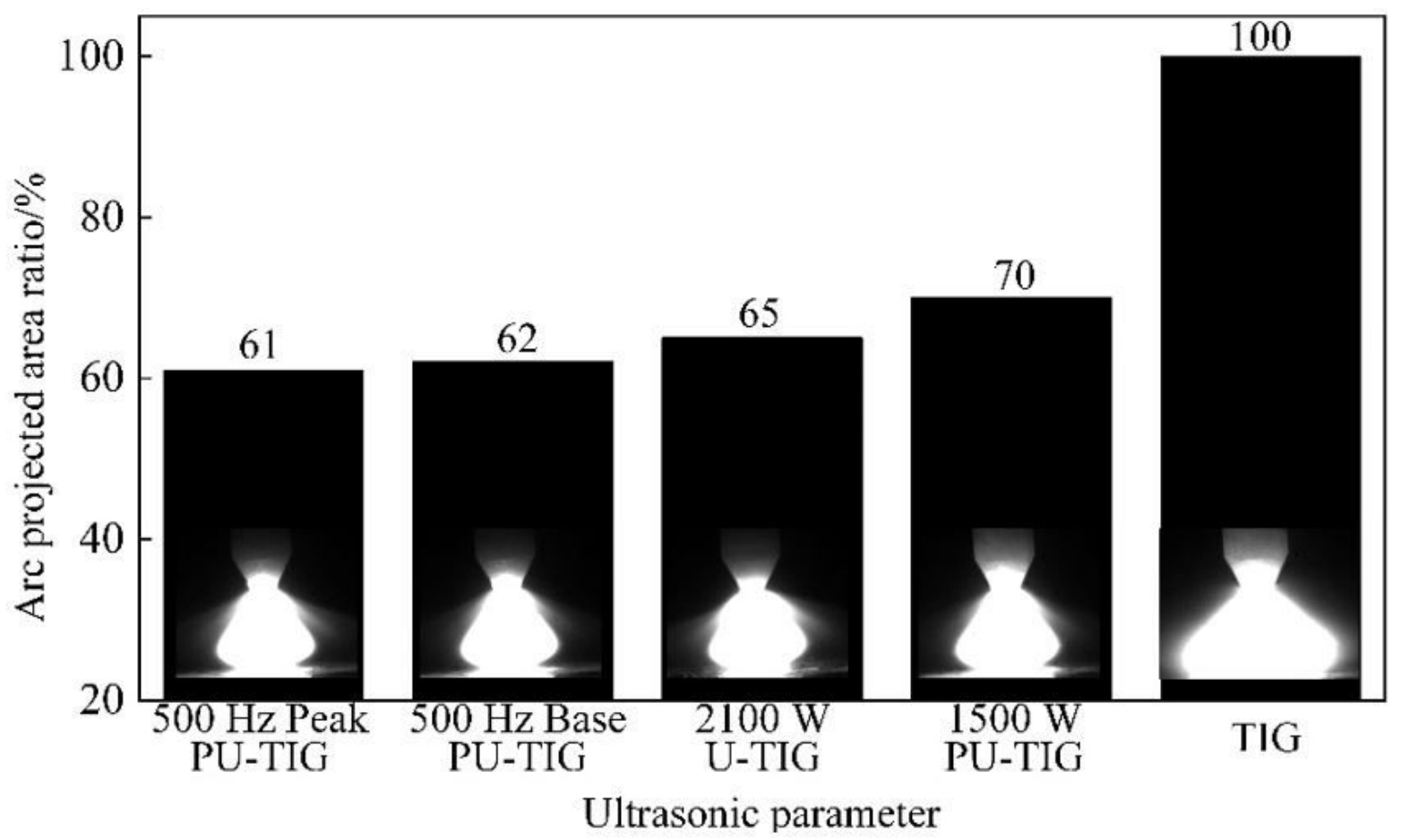

Figure 7

Arc projected area comparison. 

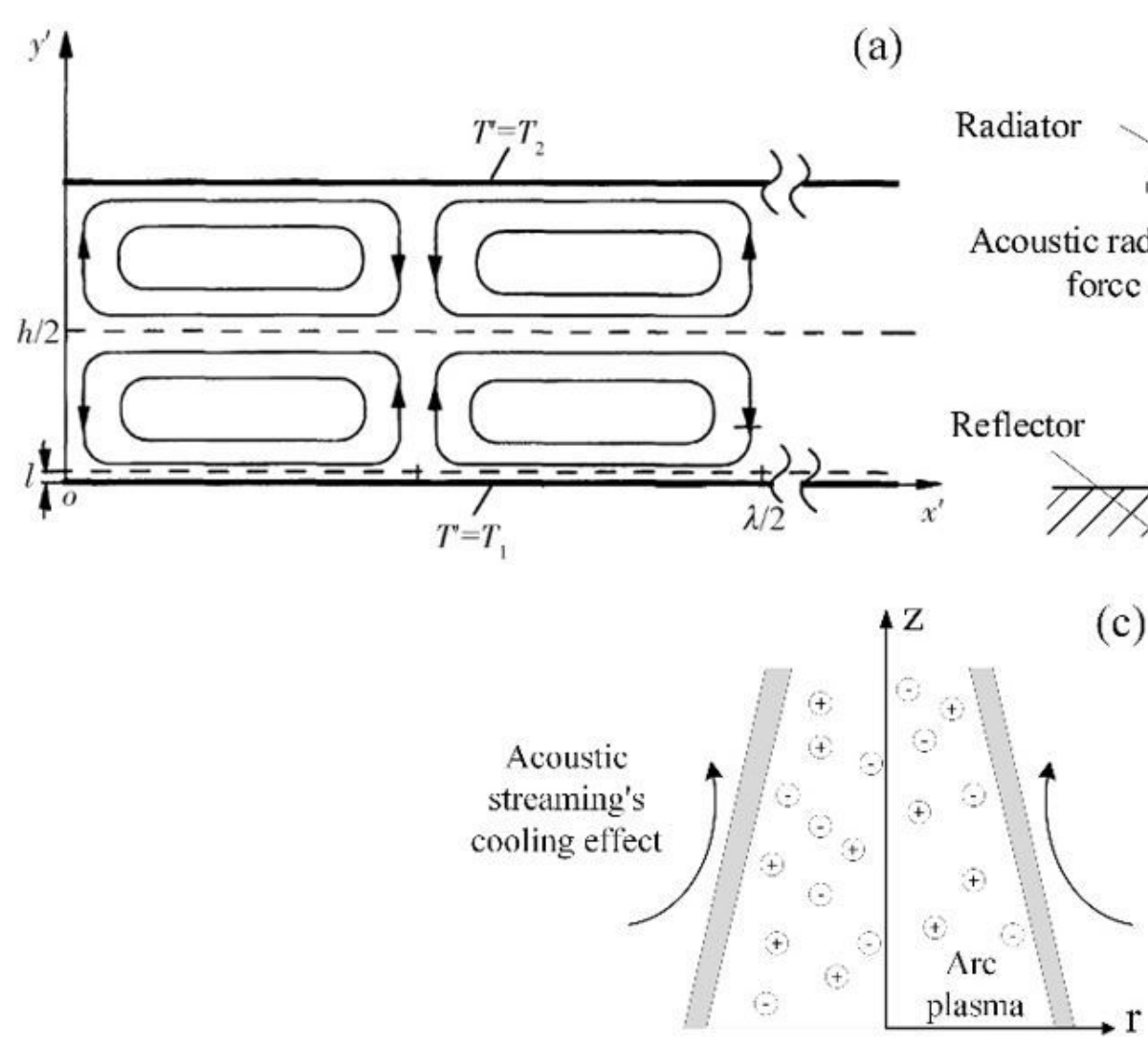

(b)

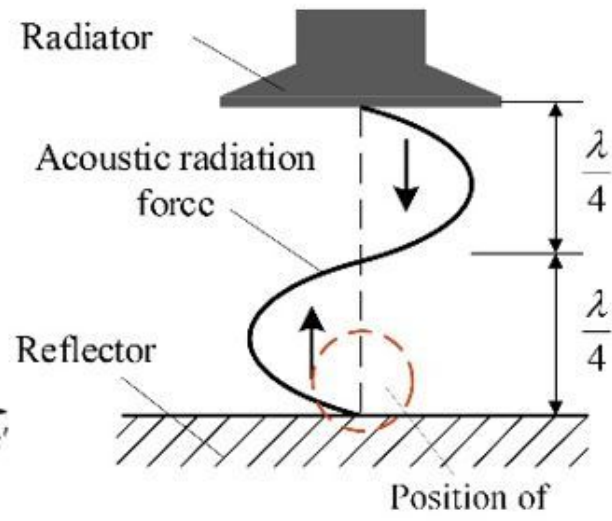

(c) arc plasma

\section{Figure 8}

Mechanism of acoustic streaming and its effect on arc. (a) Rayleigh's acoustic streaming in a standing wave field [32]; (b) distribution of acoustic radiation force; (c) cooling effect of acoustic streaming on arc;

Add continuous ultrasound of $1800 \mathrm{~W}$

Arc contraction

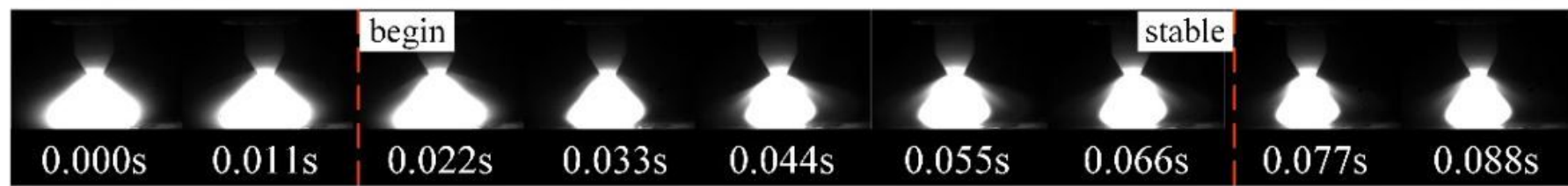

Remove ultrasound

Arc expansion

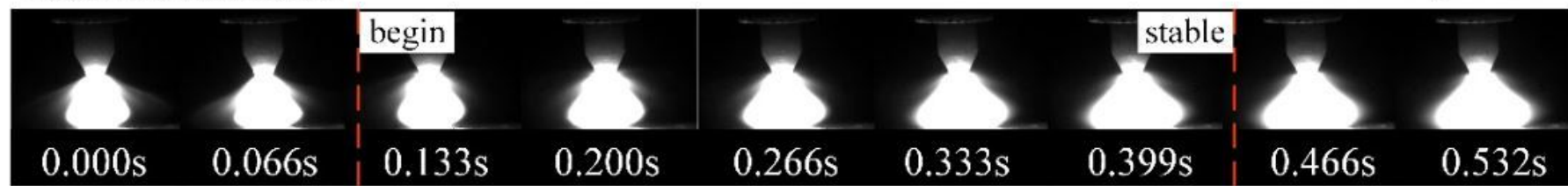

Figure 9

The speed of arc contraction and expansion 